\title{
Picture Archiving and Communication Systems and Vascular Surgery: Clinical Impressions and Suggestions for Improvement
}

\author{
Bruce I. Reiner, Eliot L. Siegel, Frank Hooper, Stephen M. Pomerantz, Zenon Protopapas, \\ Elliott Pickar, and Lois Killewich
}

\begin{abstract}
The purpose of this study was to determine the acceptance and clinical utility of a large scale picture archiving and communication system (PACS) for vascular surgery. Questionnaires and one-on-one interviews were conducted with physicians and nurses in the department of vascular surgery at the Baltimore VA Medical Center where PACS has been in routine, hospital-wide use for more than $21 / 2$ years. The perceptions of the clinical staff were assessed to determine the efficacy of PACS in comparison to the conventional film based alternative for the practice of vascular surgery and suggestions for improvements were solicited. There was consensus among the vascular surgery staff members that the use of PACS enhanced their clinical practices, both in and out of the operating room (OR). Vascular surgeons heavily rely on image display in the OR as a "road map" to help determine their operative approach and to guide their surgery. PACS offers unique intraoperative imaging capabilities including rapid image retrieval and improved archival, cine review, the ability to modify image contrast, and the ability to obtain direct quantitative measurements of the degree of vascular stenosis. The increased accessibility and availability of images throughout the hospital enables improvements in time management and in patient care.

Copyright 1996 by W.B. Saunders Company
\end{abstract}

KEY WORDS: picture archiving and communication system (PACS), radiology, angiography, vascular surgery, operating room (OR).

O NE OF THE lessons learned in the ongoing analysis of the usage of a hospitalwide picture archival and communication system (PACS) at the Baltimore VA Medical Center (BVAMC) has been that the system is used in a variety of different ways by a wide assortment of clinicians. Very little has been written concerning the effectiveness of PACS for medical and surgical subspecialties. ${ }^{1-4}$ Among the surgical subspecialties, our experience has been that the two groups that seem to rely most on diagnostic images both in and outside the operating room (OR) are the orthopedic and vascular surgery staff. The requirements of the vascular surgery department are unique in a number ways. Lost angiograms are exceptionally difficult to replace. Additionally, intraoperative angiograms can be difficult to repeat and must be made available very rapidly. Measurements of severity and length of stenosis must be made quickly and accurately. These special requirements present an interesting challenge to the capabilities of a general purpose PACS.

We describe the results of a prospective study designed to determine the degree to which a hospital-wide PACS has met these special requirements for vascular surgery and to obtain suggestions by the members of the vascular surgery staff for improvements to the system.

The BVAMC is a 300 bed teaching hospital that uses a large-scale, hospital-wide PACS (Loral Medical Imaging Systems, Chicago, IL). The system has been in continuous operation for approximately $2 \frac{1}{2}$ years. Forty-two Macintosh II or Quadra 950 workstations (Apple Computer, Cupertino, CA) are located throughout the hospital which provide access to all imaging studies which have been performed at the facility to all authorized users at any workstation. Modalities interfaced to the system include angiography, computed tomography, magnetic resonance imaging, ultrasound, nuclear medicine, fluoroscopy, general radiography using computed radiography, and the cardiac catheterization laboratory. 2,3

Workstations are distributed throughout the hospital in physician team rooms, outpatient clinics, intensive care units, the emergency room, the auditorium, the operating rooms and in the departments of radiology and nuclear medicine. Six of the eight operating rooms are equipped with a two monitor $(1,152 \times 1,078$ pixel $)$ workstation.

From the Department of Radiology and Nuclear Medicine Baltimore VA Medical Center; the Department of Radiology, University of Maryland Medical School, Baltimore; the Department of Medicine, University of Maryland Medical School, Baltimore; and the Department of Sungery, Baltimore VA Medical Center, Maryland.

Address reprint requests to Eliot $L$. Siegel, $M D, V A$ Medical Center, $10 \mathrm{~N}$ Greene St, Baltimore, MD 21201.

Copyright 1996 by W.B. Saunders Company 0897-1889/96/0903-0002\$3.00/0 
The vascular surgeons rotate between the Baltimore VA and the adjacent University of Maryland Medical System, which uses a conventional film based system. Thus they are able to evaluate the advantages and disadvantages of the digital system in comparison to the film based alternative at the University of Maryland Hospital. The nurses, however, work only at the Baltimore VA.

\section{MATERIALS AND METHODS}

Fifteen members of the vascular surgery department consisting of seven physicians and eight nurses were chosen at random from the current vascular surgery/operating room staff at the BVAMC. A forty-three question survey was administered, followed by in-depth interviews.

The survey questions asked the vascular surgery staff to evaluate multiple imaging and operational parameters emphasizing the comparison between the use of PACS and film. A subset of questions required the physicians and staff members to indicate a preference of PACS, film, or no difference. When there was a preference, a score indicating the degree of preference ranging from 1 (low) to 5 (high) was solicited. These data were tabulated according to the occupation of the respondent (see Tables 1 and 2).

\section{RESULTS}

Analysis of the interviews and questionnaires showed a preference for the PACS in comparison to the use of conventional film both in and out of the operating room. PACS was preferred over film by the physician and vascular surgery staff nurses in each of the nine comparison parameters assessed (Tables 1 and 2). The physicians most strongly preferred PACS (mean score of 3.0 to 4.0 on a scale of 1 to 5) over film in amount of useful information, availability of images, image quality, and quality of patient care. Although $33 \%$ of physicians and $100 \%$ of nurses believed that the duration of operating room cases was shorter with PACS than film, this parameter had the lowest mean score $(1.5 / 5$ for physicians and 3.9/5 for nurses).

Only five of the nine comparison parameters were deemed to be relevant to the nursing staff including overall impression of image review in the OR, time spent retrieving images, image quality, availability of images, and duration of OR cases. The nurses consistently rated their level of preference for PACS in comparison to film higher (mean range 3.9 to 5 out of 5) than did the surgeons (mean range 1.5 to 4.0 out of $5)$. However, because of the relatively small
Table 1. Response According to Occupation: Physicians

\begin{tabular}{|c|c|c|c|c|}
\hline Survey Parameter & $\begin{array}{c}\% \text { With } \\
\text { Preference } \\
\text { for PACS }\end{array}$ & $\begin{array}{c}\% \text { With no } \\
\text { Preference } \\
\text { PACS vs Film* }\end{array}$ & $\begin{array}{c}\text { Median } \\
\text { Score }\end{array}$ & $\begin{array}{l}\text { Mean } \\
\text { Score }\end{array}$ \\
\hline \multicolumn{5}{|l|}{ Amount of useful } \\
\hline information & 100 & 0 & 4 & 4.0 \\
\hline \multicolumn{5}{|l|}{ Availability of } \\
\hline images & 86 & 0 & 5 & 3.7 \\
\hline Image quality & 71 & 29 & 4 & 3.0 \\
\hline \multicolumn{5}{|l|}{ Quality of patient } \\
\hline care & 71 & 29 & 4 & 3.0 \\
\hline $\begin{array}{r}\text { Time spent } \\
\text { retrieving }\end{array}$ & & & & \\
\hline images & 86 & 0 & 3.5 & 2.9 \\
\hline $\begin{array}{l}\text { Overall impres- } \\
\text { sion of image } \\
\text { review in the }\end{array}$ & & & & \\
\hline OR & 67 & 33 & 4 & 2.8 \\
\hline \multicolumn{5}{|l|}{ Time required to } \\
\hline $\begin{array}{l}\text { Confidence in } \\
\text { making diag- } \\
\text { nosis }\end{array}$ & 57 & 43 & 4.5 & 2.6 \\
\hline \multicolumn{5}{|l|}{ Duration of OR } \\
\hline cases & 33 & 67 & 0 & 1.5 \\
\hline
\end{tabular}

*All physicians preferred PACS to film or believed there was no difference except for one of the seven vascular surgeons who rated image availability and time to retrieve studies as mildly $(2 / 5)$ better with film.

numbers of persons surveyed, the differences were not statistically significant. The physician data was somewhat skewed by a single surgeon who rated image availability and time to retrieve studies as mildly $(2 / 5)$ better with film, "amount of useful information" as mildly (2/5) better with PACS, and the other six parameters as "no preference." With the exception of this individual, the differences between the physician and nurse staff members would have been very minor. There was no significant difference in preference for PACS or film when comparing more experienced users with those less experienced with the PACS.

As was evident from the surveys, the most

Table 2. Response According to Occupation: Nurses

\begin{tabular}{cccc}
\hline Survey Parameter & $\begin{array}{c}\text { \% With Positive } \\
\text { Response }\end{array}$ & $\begin{array}{c}\text { Median } \\
\text { Score }\end{array}$ & $\begin{array}{c}\text { Mean } \\
\text { Score }\end{array}$ \\
\cline { 1 - 1 } $\begin{array}{c}\text { Overall impression of } \\
\text { image review in OR }\end{array}$ & 100 & 5 & 5.0 \\
$\begin{array}{c}\text { Time spent retrieving } \\
\text { images }\end{array}$ & 100 & 4.5 & 4.5 \\
Image quality & 100 & 4.5 & 4.2 \\
Availability of images & 100 & 4 & 4.1 \\
Duration of OR cases & 100 & 4 & 3.9 \\
\hline
\end{tabular}


commonly cited advantages of PACS in comparison to film were perceived improvements in image quality and availability because of the ability to "enhance" the images using the window/level, and magnifications and zoom capabilities of the workstations. Because of the combination of improvements in image quality and availability, the vascular surgery staff indicated that the amount of clinically useful information available with the PACS was greater than with film.

The other major advantage that was often mentioned in the interviews was the perception that the PACS provided improvements in time management and efficiency, which the surgeons considered to be particularly important to their practices. When using PACS outside of the operating room, all physicians reported substantial improvements in overall work efficiency and time management largely because of improved image accessibility throughout all locations of the hospital. The presence of PACS workstations in clinics, hospital floors, intensive care units, and workrooms resulted in rapid image retrieval and comparison during patient evaluation and work rounds. Three of seven surgeons indicated that they routinely use the PACS during a remote telephone consultation with the angiographer and discuss cases while both are simultaneously viewing the study on their workstations.

Most of the surgeons believed that the use of the PACS resulted in earlier diagnosis and treatment planning resulting in increased patient throughput and overall reduction in hospital stay. This resulted in a perceived improvement in overall patient quality of care among both physicians and nurses surveyed.

The surgeons and nurses reported that they used the window/level adjustment tool in greater than $80 \%$ of the cases and that they also frequently used the zoom and magnification tools at the workstation. Only three out of seven surgeons indicated that they used the cine tool to display sequential images rapidly. These same three physicians were the only ones that reported using the distance measurement capabilities of the workstation. The majority of the nursing staff indicated that they infrequently used these workstation tools suggesting that this was because of a "lack of confidence and knowledge of computer skills." Despite the 1 hour formal training course, two nurses reported an inability to operate the PACS other than "turning it on and off." These nurses expressed a strong interest in further education with additional formal training sessions.

Of the many modalities available on the PACS (including magnetic resonance imaging, computed tomography, nuclear medicine, cardiac catheterization, ultrasound, mammography, fluoroscopy, angiography, computed radiography $[C R]$ ), the surgeons believed that $C R$ and angiograms were the studies that were most positively affected by the PACS.

Two additional advantages of PACS identified in the surveys and interviews were a decrease in film retake rates and decreased image turnaround time for intraoperative angiograms. The average length of a vascular surgery case was reported to be approximately 4 hours, often including intraoperative angiography. Based on subjective estimates, approximately 20 minutes in time reduction for intraoperative angiography was achieved with the implementation of PACS. This reduction in the duration of surgical procedures along with improved image accessibility has led to a perceived reduction in stress levels during surgery by nurses and surgeons. The surgeons emphasized the critical importance of imaging studies, particularly angiograms, as "road maps" to help determine an optimal operative approach and to guide their surgery.

Although the surgeons were pleased in general with the ability to retrieve most images rapidly both in and outside the operating room, they did report occasional delays in image retrieval, particularly during the early phases of PACS implementation. These delays were usually due to attempts to retrieve those imaging studies that were more than 2 weeks old, had not been retrieved within the past several days, and had not been followed by a more recent study. These examinations were not stored on the central server but had to be fetched by the workstation from the optical jukebox, a process that often required from 1 to 10 minutes or more if there was already a queue of other studies for retrieval.

Both nurse and physician members of the vascular surgery staff indicated that they were 
dissatisfied with the fact that monitors were located too far from the OR tables and the lack of ability to easily move the workstation monitors that were situated on a table that was itself difficult to move. The nurses reported that the fixed location of the workstations and monitors made them "poorly accessible" to the surgical team often requiring them to leave the "operative field" and even to "break scrub" for image review. The lack of a remote control or voice activated controls were also cited as disadvantages of the current system.

The surgeons reported an overall reduction in the frequency of radiologist consultations for conventional radiographs (CR studies) because of increased image accessibility in the clinics and wards and improved report turnaround time. They did not believe that there was a corresponding reduction in the rate of consultations with the angiographers about angiographic studies with the PACS in comparison to film. The ability to consult by telephone while angiographer and surgeon are viewing the same images was seen as a major advantage of the PACS.

\section{DISCUSSION}

The PACS at the BVAMC has been well received by the members of the department of vascular surgery as has been reported for the medical housestaff ${ }^{5}$ and the surgeons in general.' Additionally, vascular surgeons and their staff members also indicated a high degree of satisfaction with the image quality and availability.

The fact that there was a moderately greater (but not statistically significant) difference in preference for the PACS in comparison to film for the nurses than for the physicians is likely due to several factors. All of the nurses had been formally training, whereas none of the surgeons had taken the training course. Thus the nurses had been given a more comprehensive overview of the capabilities of the system. Alternatively, the nurses were less likely to be called on to retrieve current and previous examinations and to use the image enhancement tools. They were thus less likely to experience some of the limitations of the current workstation software. Additionally, the nurses arguably had fewer responsibilities during the case than the surgeons and were more able to temporarily "break scrub."

The perceived improvements in image availability and efficiency suggest economic benefits of PACS in addition to savings in film costs and technical and clerical personnel that have been described by several authors. ${ }^{6-8}$ Additionally, other more difficult to measure effects such as improved diagnostic accuracy, more timely diagnosis, and improved image in the marketplace may also result in substantial savings with a hospital-wide PACS. For example, the PACS can be used enable a vascular surgeon to consult with an angiographer to decide whether a patient who is having a diagnostic angiogram should also undergo an angioplasty. This would prevent the patient from undergoing two separate procedures and could save a substantial amount of time for both physicians. Although some of the surgeons suggested that the use of the PACS might decrease the length of stay as has been suggested by some authors, ${ }^{2}$ it has been difficult to show the contribution, if any, of the PACS to the length of stay at our institution.

The fact that the majority of the surgeons did not routinely use the measurement or cine workstation tools suggests that either formal training or a "refresher course" in the operating room or vascular surgery clinic might be of value. After this additional training, a reassessment of the use of the workstation tools should be performed.

It is not surprising that the vascular surgeons and their staff believed that angiography and CR were the modalities that were most positively affected by the PACS because those are the studies most often ordered and reviewed by members of this subspecialty. The surgeons reported difficulty with manipulation of window/ level settings and image navigation for crosssectional modalities. This probably represents a combination of lack of training and the lack of window/level presets for the workstation in the operating rooms. Both of these issues will be addressed with additional training and new software that permits users to define window/ level settings specific to the user rather than a specific workstation.

The decrease in retakes and faster turnaround time for intraoperative angiograms and 
the decreased image loss rate were all major advantages of the PACS for vascular surgeons in the operating room.

There are several possible design modifications that could address the dissatisfaction with the fixed location of the two monitor workstation in the operating rooms. ${ }^{10}$ Placement of monitors on articulated arms suspended from the ceiling would permit added flexibility with respect to height and distance from the surgical field. Additionally, implementation of remote control capabilities, with sterile coverings, would permit surgeons to have direct access to the workstations without having to "break scrub" or leave the surgical field. Voice activated controls might be a viable alternative although the surgeons indicated that background noise is often relatively high in the $\mathrm{OR}$, which might make a voice activated system less reliable.

The decreased frequency of consultations for general radiographic studies reported by the vascular surgeons (but not angiograms) has been documented in other areas of the medical center. ${ }^{5,11}$ Additional conferences and frequent rounds with the vascular surgeons have been proposed to increase the interaction between radiologists and surgeons for these imaging studies. A telephone access, digital dictation system would be helpful to give vascular surgery staff immediate access to dictated reports.

In conclusion, the experience of the vascular surgeons and their nursing staff with the use of a large scale PACS has been very encouraging. As the vascular surgery department has adapted to the use of this particular PACS, so must the designers and implementers of the PACS become aware of the unique expectations and requirements of the vascular surgeons and nurses. This learning process will continue at our institution with further studies, which will supplement these subjective questionnaires and surveys with direct observations and objective measurements in addition to continued refinements of the PACS.

\section{REFERENCES}

1. Pomerantz SM, Siegel EL, Pickar E, et al: PACS in the Operating Room: Experience at the Baltimore VA Medical Center. Proceedings of IMACS Conference August 1995 (in publication)

2. Siegel EL, Diaconis JN, Pomerantz SM, et al: Making Filmless Radiology Work. J Digit Imaging 8:151-155, 1995

3. Siegel EL: Plunging into PACS. Diagn Imaging 15:6971,1993

4. Gray JE, Karsell PR, Becker GP, et al: Total Digital Radiology: Is it Feasible? Is it Desirable? AJR Am J Roentgenol 143:1345-1349, 1984

5. Siegel ES: Clinician Acceptance of PACS at the Baltimore VA Medical Center, RSNA paper presentation, November 29, 1995

6. Saarinen AO, Haynor DR, Loop JW, et al: Modeling the Economics of PACS: What is important? Proc SPIE 1093:62-73, 1989

7. Mun SK, Horii S, Benson H, et al: Experience with
Image Management Networks at Three Universities: Is the Cup Half-Empty or Half-Full? Proc SPIE 1093:194-201, 1989

8. Straub WH, Gur D: The Hidden Costs of Delayed Access to Diagnostic Imaging Information: Impact on PACS Implementation. AJR Am J Roentgenol 155:613616,1990

9. Hilsenrath PE, Smith WL, Berbaum KS, et al: Analysis of the Cost-Effectiveness of PACS. AJR Am J Roentgenol 156:177-180, 1991

10. Horii SC: Electronic Imaging Workstation: Ergonomic Issues and the User Interface. RadioGraphics 12:773787, 1992

11. Reiner BI, Siegel EL, Pomerantz SM, et al: The Impact of Filmless Radiology on the Frequency of Clinician Consultations with Radiologists. ARRS annual meeting, San Diego, CA, May 5-10, 1996 